\title{
Management Dilemma in Olanzapine Induced Restlessness and Cramps in Legs
}

\author{
Sujita Kumar Kar, Amit Singh \\ Department of Psychiatry, King George's Medical University, Lucknow, India
}

\section{TO THE EDITOR}

Restlessness and cramps in legs is a relatively rare phenomenon with antipsychotic olanzapine. If not evaluated, it may cause misdiagnosis or poor treatment adherence. ${ }^{1)}$ There is no standard guideline for management of olanzapine induced restlessness and cramps in legs, though benzodiazepines and pro-dopaminergic agents are found to be effective. $^{2)}$

A 20 year-old male had delusions of persecution, third person auditory hallucinations, inappropriate smiling with fluctuating catatonic symptoms (mutism, negativism, posturing, and rigidity) for eight months. Earlier, he was diagnosed with schizophrenia and was receiving olanzapine $10 \mathrm{mg} /$ day since three months. Intravenous lorazepam and electroconvulsive therapy was given to which catatonic symptoms responded. As there was persistence of delusion and hallucinations, olanzapine was increased to $15 \mathrm{mg}$ /day, bedtime. Patient reported cramps in both legs resulting in restless leg movements, 2-3 hours following ingestion of olanzapine in night. To get rid of discomfort and cramps in legs, he used to move his legs and even pound it over the bed desperately. The cramps would persist for 1-2 hours, causing sleep disturbance. As delusions and hallucinations were improved after increasing olanzapine, patient was maintained on same anti-psychotic dose. Management of restlessness and cramps in legs, were attempted with analgesics (started with acetaminophen $500 \mathrm{mg}$, when not responded tramadol $50 \mathrm{mg}$ had also been tried); however, it persisted causing significant disturbance of sleep. Thus the dosing of olanzapine was rescheduled to morning time. But the patient

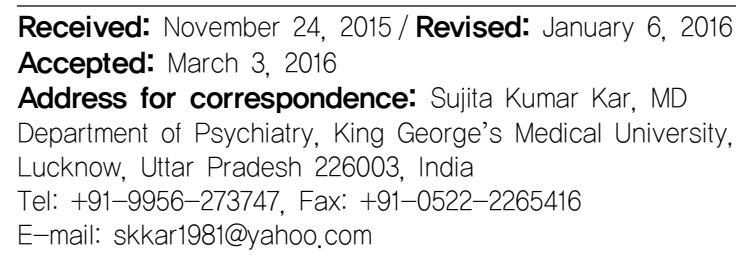

complained of restlessness and cramps in legs even during the daytime, 2-3 hours following intake of olanzapine. After this olanzapine was given in divided doses $(7.5 \mathrm{mg}$ twice a day); still the patient was complaining of restlessness and cramps in legs. Reduction of dose of olanzapine reduced the severity of leg cramps, but worsening of psychotic symptoms occurred. Patient was investigated for systemic causes that might attribute to restlessness and cramps in legs. All hematological investigation parameters (complete blood count, general blood picture, liver function test, kidney function test, thyroid function test, serum electrolytes, lipid profile as well as blood sugar) and magnetic resonance imaging of brain were within normal limits. Olanzapine was stopped and the patient was shifted to risperidone (started with $2 \mathrm{mg}$ /day and gradually built up to $6 \mathrm{mg}$ /day over a week). The patient was evaluated on Naranjo adverse drug reaction probability scale (score of 5) which was suggestive of probable adverse effect of olanzapine.

In this case, management of olanzapine induced restlessness and cramps in legs was a challenge as the patient was already receiving lorazepam $(6 \mathrm{mg} /$ day $)$ and administration of pro-dopaminergic agent (ropinirole, pramipexole or levodopa) was likely to flare up psychotic symptoms. Propranolol may have some role in reducing the restlessness, however we did not tried it, as the patient had poor oral intake due to catatonia and his vital parameters were just stable with naso-gastric feeding and parenteral fluid. Rescheduling the dose was also ineffective, hence switching to a different antipsychotic drug (risperidone) was done. As switching to risperidone is effective in olanzapine induced restlessness and cramps in legs. ${ }^{3)}$

When options are limited, switching to another antipsychotic or dose reduction may be the answer to olanzapine induced restlessness and cramps in legs.

(a) This is an Open-Access article distributed under the terms of the Creative Commons Attribution Non-Commercial License (http://creativecommons.org/licenses/by-nc/4.0) which permits unrestricted non-commercial use, distribution, and reproduction in any medium, provided the original work is properly cited. 


\section{REFERENCES}

1. Kang SG, Lee HJ, Kim L. Restless legs syndrome and periodic limb movements during sleep probably associated with olanzapine. J Psychopharmacol 2009;23:597-601.

2. Zhao M, Geng T, Qiao L, Zhang M, Shi J, Huang F, et al.
Olanzapine-induced restless legs syndrome. J Clin Neurosci 2014:21:1622-1625.

3. Basu A, Kundu S, Khurana H. Olanzapine-induced restless leg syndrome: a case report and review of literature. Indian $J$ Pharmacol 2014;46:450-452. 